\title{
Implications of Invalid Conversions between Crystal-Field Parameters and Zero-Field Splitting Ones Used in Superposition Model
}

\author{
C. RUdOWICZ ${ }^{a, *}$ AND M. KARBOWIAK ${ }^{b}$ \\ ${ }^{a}$ Institute of Physics, West Pomeranian University of Technology, al. Piastów 17, 70-310 Szczecin, Poland \\ ${ }^{b}$ Faculty of Chemistry, University of Wrocław, F. Joliot-Curie 14, 50-383 Wrocław, Poland
}

(Received February 17, 2014)

\begin{abstract}
The methodology used in recent study of the zero-field splitting parameters of $\mathrm{Cr}^{3+}$ ions at various orthorhombic symmetry sites in $\mathrm{LiKSO}_{4}$ by Pandey and Kripal is critically commented on. We argue that the crystal field parameters, $B_{k q}$, in the Wybourne notation, which were calculated using the superposition model for $\mathrm{Cr}^{3+}$ ions in $\mathrm{LiKSO}_{4}$, may only be converted into the crystal field parameters in the Stevens notation. Regrettably, the authors have also converted the latter parameters supposedly into the zero-field splitting parameters $D$ and $E$ in the conventional notation. Such direct conversions are fundamentally incorrect and constitute factual invalid usage of the conversion relations between the crystal field (ligand field) parameters and the zero-field splitting ones. The cases of an implied usage of the invalid conversion relations between the crystal field parameters and the zero-field splitting parameters occurring in recent literature are also outlined. Pandey and Kripal have found the zero-field splitting parameters theoretically evaluated in this way to be in good agreement with the experimental values. However, the faulty methodology renders the conclusion that $\mathrm{Cr}^{3+}$ ions enter into the $\mathrm{LiKSO}_{4}$ lattice at the substitutional $\mathrm{K}^{+}$sites unjustified. Several other conceptual problems arising from misinterpretations of the crucial notions identified therein are also discussed and clarified.
\end{abstract}

DOI: 10.12693/APhysPolA.125.1215

PACS: 76.30.-v, 76.30.Fc, 71.70.Ch, 75.10.Dg

\section{Introduction}

In order to elucidate the conceptual problems dealt with in this paper, a clear distinction is indispensable between the crystal field $(\mathrm{CF})$, i.e. equivalently ligand field $(\mathrm{LF})$, Hamiltonians, $H_{\mathrm{CF}}\left(H_{\mathrm{LF}}\right)$, which are fundamental in optical spectroscopy, see, e.g. [1-4], and the effective spin Hamiltonians $(\mathrm{SH}), \tilde{H}_{\mathrm{SH}}$, for a review see $[5,6]$, which are fundamental in electron magnetic resonance (EMR), see, e.g. [7-10], and magnetism, see, e.g. [11-13]. The Hamiltonians $\tilde{H}_{\mathrm{SH}}$ include two major terms, i.e. the zero-field splitting (ZFS) Hamiltonians, $\tilde{H}_{\mathrm{ZFS}}$, and the Zeeman electronic $(\mathrm{Ze})$ ones, $\tilde{H}_{\mathrm{Ze}}$. In the course of our work on the recent review [14] concerning single transition ions as well as exchange coupled systems, we have identified a variety of problems that occur at the interface between the two types of Hamiltonians: $H_{\mathrm{CF}}\left(H_{\mathrm{LF}}\right)$ and $\tilde{H}_{\mathrm{SH}}\left(\tilde{H}_{\mathrm{ZFS}}\right)$.

The problem that is most pertinent for the present considerations is the CF = ZFS confusion, which is defined as the cases of labeling the true ZFS quantities as purportedly the CF (LF) quantities. Various types of terminological confusions between the CF (LF), SH (ZFS), and related quantities, identified so far in literature have been discussed in the reviews $[5,6,15]$ and most recently [14]. For the background theory and notations,

\footnotetext{
*corresponding author; e-mail: crudowicz@zut.edu.pl
}

which illustrate the actual nature of the interrelationships between the CF parameters (CFPs) and the ZFS parameters (ZFSPs) and enable subsequent clarifications, the readers are advised to consult the review [14]. To provide perspective, in Sect. 2 we outline briefly the cases of an implied usage of the invalid conversion relations between the CFPs and the ZFSPs occurring in recent literature. The faulty methodology, which constitutes factual invalid usage of such conversion relations, and several conceptual problems identified in the study [16] of the ZFSPs of $\mathrm{Cr}^{3+}$ ions at various orthorhombic symmetry sites in lithium potassium sulphate, $\mathrm{LiKSO}_{4}$, are critically commented on in Sect. 3 .

\section{Implied usage of the invalid conversion relations between the $\mathrm{CF}$ parameters and the ZFS ones}

It is essential to bring to the attention of the readers the major points elucidated in the review [14]. There are two major types of operators that used to express the CF (LF) and SH (ZFS) Hamiltonians, i.e. the Stevens and Wybourne ones. These operators have distinct properties, which have been classified and discussed in detail in [14] together with several key aspects. This has included: basic forms of Hamiltonians and definitions of the associated parameters, distinct properties of the Stevens and Wybourne CF (LF) parameters and implications for conversion relations, distinctions and interrelationships between the CF (LF) and SH (ZFS) Hamiltonians and 
parameters, conversion relations for the Stevens and conventional ZFS parameters, and orthorhombic standardization.

Specific types of serious terminological confusion concerning the notions of $H_{\mathrm{CF}}\left(H_{\mathrm{LF}}\right)$ and $\tilde{H}_{\mathrm{SH}}\left(\tilde{H}_{\mathrm{ZFS}}\right)$ bear on the conversion relations suitable for the CF (LF) parameters and the ZFS parameters [14]. One type of the $\mathrm{CF}=\mathrm{ZFS}$ confusion has led to an implied usage of the invalid conversion relations between the CF (LF) parameters and the ZFS ones. By implied usage, we mean providing such relations and considering them as physically correct but not followed by any numerical calculations. Examples of such implied usage have been identified, e.g. in the recent tutorial review by Sorace et al. [17] of the magnetic properties of single molecule magnets (SMMs) based on the rare-earth ions. As discussed [14], the authors [17] have provided in their eq. (5) the conversion relations supposedly for the ZFSPs expressed in the Stevens and conventional notations (for definitions see the reviews $[5,6])$. Equation (5) in [17] was presented as relations between "the elements of $D$ ", i.e. the ZFS tensor $D_{i j}$, and "the $B_{k}^{q}=\beta_{k} A_{k}^{q}\left\langle r^{k}\right\rangle$ set of parameters". Incidentally, it has turned out that the relations [17] were misprinted - for details see [14], whereas the correct relations of this type may be found in [18-20].

More importantly, the relations given in [17] are misleading since they imply their applicability for the cross-conversions between the CF (LF) parameters expressed in the Stevens notation, i.e. $B_{k}^{q}=\beta_{k} A_{k}^{q}\left\langle r^{k}\right\rangle$, and the ZFS parameters, i.e. $D_{i j}$, expressed in the conventional notation. In fact, such relations apply strictly only for conversions between the ZFS parameters expressed in the Stevens notation, $B_{k}^{q}$ (or $b_{k}^{q}$ ), and those expressed in the conventional notation, $D_{i j}$ - for definitions and nomenclature see $[5,6]$. Hence, the usage of the conversions relations implied in the review [17] is fundamentally invalid, since for the reasons discussed in [14] these relations shall never be employed for conversions between any ZFSPs nor for cross-conversions between the CFPs and the ZFSPs. Let us note that the same symbol $B_{k}^{q}$ may denote either the CFPs or the ZFSPs, however, the notation $B_{k}^{q}=\beta_{k} A_{k}^{q}\left\langle r^{k}\right\rangle$ is applicable exclusively for the CFPs, whereas makes no sense for the ZFSPs.

Apart from the review by Sorace et al. [17], an implied usage of the invalid conversion relations has also been identified [14] in other recent papers, namely, Yamashita et al. [21] on magnetic anisotropy of $\mathrm{Ln}^{\mathrm{III}}$-based SMMs, the User Manual for the computer program Phi developed by Chilton [22], and the review of magnetic polyoxometalates by Clemente-Juan et al. [23]. Similar pitfall occurs also in the papers utilizing the software package SIMPRE developed by Baldoví et al. [24]. Importantly, a relation to convert the ZFS parameters into the CF (LF) ones has been practically utilized in the papers $[25,26]$ for the purpose of qualitative conclusions. As discussed in [14], this pitfall has led also to inappropriate interpretation of the results [24-26]. However, no factual invalid usage of such conversion relations, i.e. ap- plications for numerical recalculations, has taken place in the papers $[17,21-26]$. The confusion concerning the questionable relations in $[17,21-26]$ and similar ones appearing in the earlier literature (for references, see [14]) have originated most probably from careless notation and wording, whereas rather not from a real misunderstanding by the respective authors.

The readers should beware that any attempt to employ such relations to convert a concrete set of the CFPs into the ZFSPs or other way round would be fundamentally incorrect and hence would lead to wrong numerical results. Unfortunately, such conversion relations have independently been utilized in a recent paper by Pandey and Kripal [16]. In Sect. 3 we comment on this serious case, which constitutes factual invalid usage of the conversion relations between the CF ( $\mathrm{LF}$ ) parameters and the ZFS ones, and discuss its implications.

\section{Factual usage of the invalid conversion relations between the CFPs and the ZFSPs ones and its implications}

The direct conversions between the CFPs and the ZFSPs utilized in [16] constitute faulty methodology, which has lead to seriously flawed calculations of the ZFSPs $D$ and $E$ for $\mathrm{Cr}^{3+}$ ions in lithium potassium sulphate $\mathrm{LiKSO}_{4}$. This has made the conclusions [16] groundless and most probably invalid. The procedure used by the authors [16] is briefly outlined and several pertinent problems are exposed below.

The Hamiltonians used [16] were defined as follows: $H_{\mathrm{CF}}$ as equivalent to eq. (4) and expressed in terms of the CFPs $B_{k q}$ and "the Wybourne spherical tensor operators" $C_{q}^{(k)}$, whereas $\tilde{H}_{\mathrm{ZFS}}$ as equivalent to eq. (9) with the ZFSPs: $D$ (axial) and $E$ (rhombic). The CFPs $B_{k q}$ were calculated based on the crystallographic data and using superposition model (SPM) - for references, see the reviews [27-30]. Model parameters specific for SPM/ CFP calculations were employed. It is important to keep in mind that SPM may be used independently also for SPM/ZFSP calculations, however, then different sets of model parameters specific for ZFSPs must be employed as discussed below.

The most controversial statements [16] are, quote: "Considering the transformation properties of the Stevens operators $O_{k}^{q}$, the relations between the arbitrary symmetry SH parameters and CFPs in the Stevens notation $B_{k}^{q}$ were derived in various axis systems [28] (i.e. Ref. [31] in this paper). Using consistent convention prevailing in the recent literature we have the following relations between the conventional ZFS parameters $D$ and $E$ and CF parameters $B_{k}^{q}$ :

$$
D=3 B_{2}^{0}, \quad E=B_{2}^{2} \text {. }
$$

Taking these relations and the idea that the ratio $E / D$ for an orthorhombic symmetry Hamiltonian can always be limited to the range $(0, \pm 1 / 3)$, the conventional $D$ and $E$ are calculated by obtaining $B_{k}^{q}$ from $B_{k q}$ (CFPs 
in the Wybourne notation), where $B_{k q}$ are determined from the $[\mathrm{SPM}]$ expressions given in Appendix A."

Major problems evident in the above quotes are as follows.

(1) Equation (3) in [16] quoted above represents only the higher symmetry (axial and rhombic) ZFSPs appearing in the full set of relations applicable to triclinic symmetry cases [14, 17]. However, the interpretation of such relations provided in [16] as suitable for conversions between "conventional ZFS parameters $D$ and $E$ and CF pa-

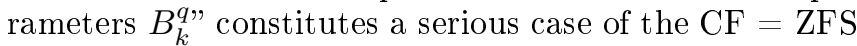
confusion. This conclusion applies also to the similar interpretation provided in [17]. As explained in [14], such equations do not relate the CFPs with the ZFSPs; they only merely convert the ZFSPs from the Stevens notation to the conventional one and vice versa.

(2) The authors [16] have failed to realize the consequences of the fact that for $\mathrm{Cr}^{3+}\left(3 d^{3}, S=3 / 2\right)$ ions the parameters of the rank $k=2$ and 4 exist for CFPs, whereas only of the rank $k=2$ for ZFSPs. Hence, it is even not technically feasible to convert the fourth-rank CFPs to the corresponding ZFSPs. Had the authors [16] realized this fact, they could have been warned about the incorrectness of the procedure used.

(3) The fact invoked in [16] that the rhombicity ratio of the conventional ZFSPs $\lambda=E / D$ may be always limited to the range $(0,1 / 3)$ pertains implicitly to the orthorhombic standardization introduced in [31]. This standardization has been widely applied both for the ZFSPs and the CFPs - for references, see [32-36]. However, in this context there is no point to invoke the orthorhombic standardization, since this idea has nothing to do with the presumed conversions of the parameters. Moreover, there is no indication in [16] that orthorhombic standardization has been actually utilized and for which parameter sets. Instead, the conversion relations actually used for "obtaining $B_{k}^{q}$ from $B_{k q}$ " (see [14]) should have been provided either explicitly or by citing pertinent references.

Concerning the procedure used in [16] the following comments are pertinent. The authors [16] have first performed conversion of the CFPs calculated using SPM/ CFP and expressed in the Wybourne notation, $B_{k q}$, into the CFPs expressed in the Stevens notation, $B_{k}^{q}(\mathrm{CF})$. This step is perfectly valid. However, at the next step, based on the misinterpretation in the point (1) above see also [14], the later values, i.e. $B_{k}^{q}(\mathrm{CF})$, have been used incorrectly to obtain, via eq. (3) in [16] quoted above, the supposedly theoretically evaluated $B_{k}^{q}(\mathrm{ZFS})$. Several distortion models for $\mathrm{Cr}^{3+}$ ions at interstitial site and substitutional sites in $\mathrm{LiKSO}_{4}$ have been attempted. For example, in the first attempt for $\mathrm{Cr}^{3+}$ at interstitial site the so obtained [16] CFPs yield " $D$ and $E$ to be $11241.7 \mathrm{~cm}^{-1}$ and $21586.8 \mathrm{~cm}^{-1}$, which are inconsistent with the experimental values". The shear difference in the magnitude between these calculated values and the experimental ZFSPs $D$ and $E$ (see above) should have stricken a note of caution about the procedure used. Im- portantly, such curiously disparate results have been obtained for all other distortion models considered, except the final supposedly satisfactory attempt. By assuming that $\mathrm{Cr}^{3+}$ ion occupies substitutional $\mathrm{K}^{+}$site in $\mathrm{LiKSO}_{4}$ and manipulating the values of the model parameters for the SPM/CFP calculations to match the calculated and experimental ZFSPs $D$ and $E$, a "reasonable agreement with the experimental values" has been obtained. However, neither the matched values of the model parameters nor the conclusion that "the results suggest that $\mathrm{Cr}^{3+}$ ion occupies substitutional $\mathrm{K}^{+}$site in $\mathrm{LiKSO}_{4}$ " may be considered as meaningful in view of the faulty and unacceptable procedure used.

Other doubtful problems may be mentioned. We note that the notion of the microscopic spin Hamiltonian (MSH) theory as used in [16] has been misinterpreted. The authors [16] state that, quote: "The zero field splitting parameters $D$ and $E$ are then determined using microscopic spin Hamiltonian theory and compared with the experimental values obtained by electron paramagnetic resonance." and "The EPR ZFS parameters have been investigated using the superposition model and microscopic spin Hamiltonian theory." Contrary to these claims, in fact, no MSH theory has been utilized but only the faulty methodology outlined above. As discussed at a conceptual level in [14], MSH theory, historically [7-12] pertains to an explicit derivation of $\tilde{H}_{\mathrm{SH}}$ for single transition ions with an orbital singlet ground state by methods based on perturbation theory — for a review, see $[5,6]$.

Another problem concerns the maximum rhombicity, i.e. $\lambda=E / D=1 / 3$, which is admissible by the standardization [31] of orthorhombic symmetry $\tilde{H}_{\mathrm{ZFS}}$ and is also applicable to $H_{\mathrm{CF}}$, see, e.g. [31-36]. The ratio of the final calculated [16] ZFSPs $E / D=0.32$ is close to the maximum value $1 / 3$, whereas the experimental values obtained earlier using EPR: [37] 549 and 183 in $\left[10^{-4} \mathrm{~cm}^{-1}\right]$, yield $E / D=1 / 3$. This casts additional doubts on the reliability of both sets of $(D, E)$ values. The SPM relations for the CFPs $B_{k q}$ were provided only for $\mathrm{Cr}^{3+}$ positioned at the $\mathrm{K}^{+}$site in $\mathrm{LiKSO}_{4}$, hence the correctness of the SPM/CFP calculations for other distortion models considered cannot be verified. Let us note that two specific problems concerning the rhombicity ratio identified in the SMM and magnetism studies, namely, (i) usage of fixed values of $\lambda$ to facilitate numerical calculations and (ii) misinterpretation of the convention $0<|\lambda| \leq 1 / 3$, have been critically discussed in [14].

Finally, doubts arise if the metal-oxygen bond distances $R_{j}$ and coordination angles $\theta_{j}$ and $\phi_{j}$ used in $\mathrm{SPM} / \mathrm{CFP}$ calculations for $\mathrm{Cr}^{3+}$ ion doped into $\mathrm{LiKSO}_{4}$ single crystals are reliable. In the description of the crystal structure of $\mathrm{LiKSO}_{4}$ the authors state, quote [16]: "The $\mathrm{Li}^{+}$ion has a tetrahedral coordination with $\mathrm{Li}-\mathrm{O}$ distances in the range $0.1909-0.1923 \mathrm{~nm}$. The $\mathrm{K}^{+}$ion is surrounded by nine $\mathrm{O}$ atoms of sulphate at distances 0.2840-0.2989 nm." However, in their Fig. 1 the environment of $\mathrm{K}^{+}$and coordination around $\mathrm{Cr}^{3+}$ in $\mathrm{LiKSO}_{4}$ represents an octahedral sixfold arrangement of the $\mathrm{O}$ 
ligands. Yet the values of $\left(R_{j}, \theta_{j}, \phi_{j}\right)$ listed in their Tables I-III when the $\mathrm{Cr}^{3+}$ ion is assumed at the case: (I) point between $\mathrm{O}(1)-\mathrm{O}(2)$ bond, (II) $\mathrm{Li}^{+}$site, and (III) $\mathrm{K}^{+}$site, contain data for four $\mathrm{Cr}-\mathrm{O}(\mathrm{i})$ bonds each . The actual coordination and local site symmetry in the case $I$ is not clear, whereas selection of "point between $\mathrm{O}(1)-\mathrm{O}(2)$ bond" has not been justified on crystallographical grounds. Importantly, the number of ligands considered in the case III (i.e. four) contradicts the description in text (i.e. nine) as well as the arrangement in their Fig. 1 (i.e. six). The latter case is crucial in view of the authors' conclusion cited above. We are not in the position to repeat and verify the reliability of the calculations in [16]. It may be expected that future joint investigations along the lines suggested in Sect. 3 may solve these doubts.

\section{Conclusions and outlook}

It may be expected that elucidation of conceptual problems arising from misinterpretations of the crucial notions exposed in this paper will stimulate readers from related fields to look more deeply into the intricacies discussed here and in the reviews $[5,6,14,15]$. This in turn may help improving understanding of these intricacies, especially among experimentalists, and thus preventing proliferation of the $\mathrm{CF}=\mathrm{ZFS}$ confusion discussed herein. We do hope that all colleagues concerned will accept our efforts not as a mere criticism but as a valid call to amend the situation before it will go out off hands.

The major problems outlined in Sect. 3 have been privately communicated to the authors [16] as a matter of courtesy by one of us (Cz.R.) and the ways for amending the mistakes have been proposed. This includes primarily better understanding of the distinction between the crystal field (ligand field) parameters (CFPs) and ZFSPs as well as of the orthorhombic standardization and the limits of valid applications of the pertinent conversion relations. Subsequently, the way out of this awkward situation has been suggested. It seems that the best option would be to redo all calculations using SPM model parameters specific not for CFPs as originally attempted [16], but for ZFSPs. The recent applications of the SPM/ZFSP approach [38-42] may serve as guidance in this regard.

To find suitable SPM model parameters specific for ZFSPs for $\mathrm{Cr}^{3+}$ ions in $\mathrm{LiKSO}_{4}$, the data accumulated in the extensive literature database (for short LDB) could be helpful. This database has been developed over the years (nearly 45) by one of the authors (Cz.R.) to facilitate research in optical and EMR spectroscopy as well as magnetism of transition ions. At present it contains nearly 20,000 records covering all major journals and spanning over 80 years. The Excel files can be searched on computer for various useful items or topics coded in appropriate columns. The full literature databases, together with the relevant explanatory files as well as subsequent updates, are available from Cz.R. upon request.
The database LDB contains at present huge number of records pertaining to applications of the SPM/ZFS as well as SPM/CF approach. The proposed new and thoroughly checked SPM calculations of CFPs may be also presented in the amended version of the paper [16]. However, it is advisable that the results should be complemented by comparison of the SPM calculated CFPs with suitable literature values to verify their validity, whereas the new and rechecked "theoretical energy values of different transitions" may be presented as in Table $\mathrm{V}$ in [16].

\section{Acknowledgments}

One of us (Cz.R.) would like to thank Prof. R. Kripal for the Visiting Professorship in his group in the period 3-21 Sept. 2007, which provided an opportunity for collaboration as well as for superb hospitality.

\section{References}

[1] B.N. Figgis, M.A. Hitchman, Ligand Field Theory and Its Applications, Wiley-VCH, New York 2000.

[2] Crystal Field Handbook, Eds. D.J. Newman, B. Ng, Cambridge University Press, Cambridge 2000.

[3] M. Wildner, M. Andrut, C. Rudowicz, in: Spectroscopic Methods in Mineralogy - EMU Notes Mineralogy, Eds. A. Beran, E. Libowitzky, Vol. 6, Eötvös Univ. Press, Budapest 2004, Ch. 3, p. 93.

[4] G. Liu, B. Jacquier, Spectroscopic Properties of Rare Earths in Optical Materials, Eds.: G. Liu, B. Jacquier, Tsinghua University Press and Springer, Berlin 2005.

[5] C. Rudowicz, Magn. Reson. Rev. 13, 1 (1987); erratum, ibid., 13, 335 (1988).

[6] C. Rudowicz, S.K. Misra, Appl. Spectrosc. Rev. 36, 11 (2001).

[7] J.A. Weil, J.R. Bolton, Electron Paramagnetic Resonance, Elemental Theory and Practical Applications, Wiley, New York 2007.

[8] J.R. Pilbrow, Transition-Ion Electron Paramagnetic Resonance, Clarendon Press, Oxford 1990.

[9] F.E. Mabbs, D. Collison, Electron Paramagnetic Resonance of $d$ Transition-Metal Compounds, Elsevier, Amsterdam 1992.

[10] Multifrequency Electron Paramagnetic Resonance, Ed. S.K. Misra, Wiley-VCH, Weinheim 2011.

[11] K.W.H. Stevens, Magnetic Ions in Crystals, Princeton Univ. Press, Princeton 1997.

[12] R. Boča, Theoretical Foundations of Molecular Magnetism, Elsevier, Amsterdam 1999.

[13] K.H.J. Buschow, F.R. de Boer, Physics of Magnetism, Magnetic Materials, Kluwer Academic, New York 2003.

[14] C. Rudowicz, M. Karbowiak, Interface between the physical crystal field Hamiltonians and the effective spin Hamiltonians, a primer for experimentalists, in preparation, 2014.

[15] C. Rudowicz, H.W.F. Sung, Physica B 337, 204 (2003).

[16] S. Pandey, R. Kripal, Acta Phys. Pol. A 123, 101 (2013). 
[17] L. Sorace, C. Benelli, D. Gatteschi, Chem. Soc. Rev. 40, 3092 (2011).

[18] T.H. Yeom, C. Rudowicz, S.H. Choh, D.G. McGavin, Physica Status Solidi B 198, 839 (1996).

[19] C. Rudowicz, P. Gnutek, Physica B 403, 2349 (2008).

[20] P. Gnutek, C. Rudowicz, Opt. Mater. 31, 391 (2008).

[21] A. Yamashita, A. Watanabe, S. Akine, T. Nabeshima, M. Nakano, T, Yamamura, T. Kajiwara, Angew. Chem. Int. Ed. 50, 4016 (2011).

[22] N.F. Chilton, PHI User Manual v1.7, 2013.

[23] J.M. Clemente-Juan, E. Coronado, A. Gaita-Arino, Chem. Soc. Rev. 41, 7464 (2012).

[24] J.J. Baldoví, S. Cardona-Serra, J.M. Clemente-Juan, E. Coronado, A. Gaita-Arino, A. Palii, J. Comput. Chem. 34, 1961 (2013).

[25] J.J. Baldoví, S. Cardona-Serra, J.M. Clemente-Juan, E. Coronado, A. Gaita-Ariño, A. Palii, Inorg. Chem. 51, 12565 (2012).

[26] J.J. Baldoví, J.J. Borrás-Almenar, J.M. ClementeJuan, E. Coronado, A. Gaita-Ariño, Dalton Trans. 41, 13705 (2012).

[27] D.J. Newman, W. Urban, Adv. Phys. 24, 793 (1975).

[28] D.J. Newman, B. Ng, Rep. Prog. Phys. 52, 699 (1989).

[29] D.J. Newman, B. Ng, in: Crystal Field Handbook, Eds. D.J. Newman, B. Ng, University Press, Cambridge 2000, Ch. 5, p. 83.

[30] M. Andrut, M. Wildner, C. Rudowicz, in: Spectroscopic Methods in Mineralogy, EMU Notes in Mineralogy, Vol. 6, Eds. A. Beran, E. Libowitzky, Eötvös University Press, Budapest 2004, Ch. 4, p. 145.
[31] C. Rudowicz, R. Bramley, J. Chem. Phys. 83, 5192 (1985).

[32] C. Rudowicz, P. Gnutek, Physica B 404, 113 (2010).

[33] C. Rudowicz, J. Qin, J. Lumin. 110, 39 (2004).

[34] R. Kripal, D. Yadav, P. Gnutek, C. Rudowicz, J. Phys. Chem. Solids 70, 827 (2009).

[35] C. Rudowicz, P. Gnutek, J. Phys., Condens. Matter 22, 45501 (2010)

[36] D. Yadav, R. Kripal, P. Gnutek, C. Rudowicz, J. Phys. Chem. Solids 74, 751 (2013).

[37] R. Kripal, I. Mishra, Mater. Chem. Phys. 119, 230 (2010).

[38] P. Gnutek, M. Açıkgöz, C. Rudowicz, Opt. Mater. 32, 1161 (2010).

[39] M. Acikgoz, P. Gnutek, C. Rudowicz, Solid State Commun. 150, 1077 (2010).

[40] M. Acikgoz, P. Gnutek, C. Rudowicz, Solid State Commun. 150, 1610 (2010).

[41] M. Açıkgöz, P. Gnutek, C. Rudowicz, Chem. Phys. Lett. 524, 49 (2012).

[42] M. Açıkgöz, P. Gnutek, C. Rudowicz, Chem. Phys. 402, 83 (2012). 